\title{
A Referenced Cyber Physical System for Compressor Manufacturing
}

\author{
Jin $\mathrm{Cao}^{1, *}$, Junliang $\mathrm{Wang}^{2}$, and Junqing $\mathrm{Lu}^{1}$ \\ ${ }^{1}$ Shanghai Highly(group) Co., Ltd, Shanghai, China \\ ${ }^{2}$ College of Mechanical Engineering, Donghua University, Shanghai, China
}

\begin{abstract}
Compressor is a typical high-end discrete product, with the shortening of product life cycle and the enhancement of the degree of product customization, the traditional compressor manufacturing system architecture cannot meet the requirements of comprehensive digital management of compressor from body scheme design to parts production line, logistics management, operation and maintenance monitoring and evaluation. This paper presents a compressor manufacturing system architecture based on digital twinning, and establishes an Internet platform for compressor industry oriented to remote coordination from three aspects of compressor design, production, operation and maintenance. The platform includes industrial Internet infrastructure layer, physical space entity model layer, virtual space multidimensional model layer, physical space and virtual space multidimensional model correlation and mapping layer, big data intelligent analysis decision-making layer, and digital twin application layer. Through the establishment of the compressor product design and simulation model of digital twin, compressor production process digital twin model, compressor fault diagnosis and remote operations digital twin model, implementation is based on the number of compressor collaboration in manufacturing industrial Internet platform twin system, leading the transformation and upgrading of intelligent manufacturing industry, compressor industry sustainable development ability and international competitiveness.
\end{abstract}

\section{Introduction}

Compressor is a typical high-end discrete product whose rotor manufacturing accuracy is as high as micron level. There are more than 1000 kinds of products and tens of thousands of parts. Therefore, compressors workshop is a typical high-end discrete manufacturing system [1]. In recent years, with the integration of new generation information technology, equipment and processes have been promoted from automation to intelligence. At present, the traditional compressor manufacturing system architecture can't meet the needs of comprehensive management from compressor design to parts production line, logistics management, operation and maintenance monitoring, and evaluation. Traditional compressors are facing enormous challenges of transformation and upgrading.

Cyber Physical System (CPS) is the key technology of the new generation of intelligent manufacturing [2]. It reflects all the characteristics of the object from micro to macro and demonstrates the evolution process of the product life cycle (PLC), Then realizes the performance optimization of the whole life cycle through building the digital mirror model of physical products and integrating with external sensors.

In the stage of compressor design, the way of combining three-dimensional digital model with twodimensional drawings is still adopted in the design scheme of compressor in China [3]. Design dimensions, process documents and test data are in the state of data island now. How to integrate multi-dimensional design data and realize intelligent design through digital twin model is the key factor to drive compressor design intellectualization.

In the process of compressor production, the data generated in the production site (operation data of instruments and equipment, production logistics data, production progress data, production personnel data, etc.) could be collected and processed in real time by digital twin technology, and the process data can be mapped and matched with the digital twins of compressor products and production line [4]. The refined management of the compressor product manufacturing process can be realized online. At the same time, it combines data mining and machine learning algorithm (cloud-based intelligent information platform, dynamic Bayesian and neural network) to realize real-time dynamic optimization and adjustment of production line, manufacturing unit, production schedule, logistics and quality.

Compressor operation and maintenance is the core of air conditioning equipment operation and maintenance, especially in new energy automotive compressor. The normal operation of compressor not only affects the car environment, but also determines the working temperature of the battery and affects the output

Corresponding author: caojin@highly.cc 
efficiency of the battery. It is the focus of affecting the operation efficiency of new energy automobiles [5]. In the process of compressor operation and maintenance, the physical space position, external environment, quality status, use status, technology and function status of compressor products are tracked and monitored in real time. The health status, life span, function and performance of compressor products are predicted and analyzed according to the actual state, real-time data, using and maintenance record data of compressor products. Meanwhile, the quality of products will be early warned. When product failures and quality problems occur, it could realize rapid positioning of product physical location, fault and quality problems record, cause analysis, parts replacement, product maintenance, product upgrade or even scrap, and decommissioning, etc.

Therefore, starting from the three aspects of compressor design, production, operation and maintenance, this paper establishes an industrial Internet platform for remote collaboration, a digital twin model for compressor product design and simulation, a digital twin model for compressor production process, and a digital twin model for compressor fault diagnosis and remote operation and maintenance to realize the digital twin system of compressor co-manufacturing in different places based on industrial Internet platform, lead the industry to transform and upgrade to intelligent manufacturing, and enhance the sustainable development ability and international competitiveness of compressor industry.

\section{Literature review}

Digital twin has received wide and high attention recently. Gartner, the most authoritative IT research and consulting firm, has listed digital twin as one of the top ten strategic technology development trends for two consecutive years (2016 and 2017). Lockheed Martin, the largest weapons manufacturer, has listed digital twin as the top six technologies in the future defense and aerospace industry in November 2017. On December 8, 2017, digital twin was listed as one of the top ten technological advances in intelligent manufacturing by the Intelligent Manufacturing Alliance of CAST Member Societies at the World Intelligent Manufacturing Congress [6][7].

In addition, many famous international enterprises have begun to explore the application of digital twin technology in product design, manufacturing and service. In product design, for innovative design of complex products, Dassault [8] has established a 3D experience platform based on digital twin, which continuously improves the product design model in the information world and feeds back to the physical entity product improvement by using users' interactive feedback information. In terms of production and manufacturing, Siemens [9] constructs the production system model of integrated manufacturing process based on digital twin concept, and forms the virtual enterprise model and the enterprise image based on automation technology to support the enterprise in integrating and digitalizing the entire value chain, and carry out application verification in the production process of Siemens industrial equipment Nanobox PC. In fault prediction and health management, NASA [10] combines physical system with its equivalent virtual system, studies the method of fault prediction and elimination of complex systems based on digital twin, and applies it to the health management of flight systems such as aircraft and launch vehicle. The Center for Structural Sciences of the Air Force Research Laboratory [11] combines the virtual model of ultra-high-fidelity aircraft with the structural deviation and temperature calculation models that affect flight and carries out the life prediction of aircraft structure based on digital twin. In terms of product service, PTC company [12] takes digital twin as the key link of 'intelligent interconnected products', and devotes itself to establishing a real-time connection between the virtual world and the real world to extend every action of intelligent products to the next product design cycle and realize predictive maintenance of products. Meanwhile, customers are provided with efficient after-sales service and support. In addition, workshop is the execution basis of manufacturing activities. In order to practice the concept of intelligent manufacturing and intelligent production, Fei Tao et al. [13][14] explored the concept of digital twin workshop, designed the composition and operation mechanism of DTS, expounded four characteristics and five key technologies of DTS, and provided basic theoretical support for the application of digital twin in production and manufacturing in 2017. Negri E et al. [15] analyzed the background of digital twin, expounded the connotation of product digital twin, and proposed the architecture and implementation approach of product digital twin. At the same time, he pointed out that the emergence and development of digital twin technology provided not only a new idea, method and implementation approach for the realization of Information Physics System, but also new ideas and tools for human to explore and predict the unknown world with virtual model. Schleich B [16] and others discussed the application of digital twin model in product configuration management. Zhang et al. [17] proposed a rapid customization design method for personalized production line based on digital twin, which synchronized the virtual and real of digital model and physical equipment to form a whole line digital twin system. He proposed a decoupling algorithm for coupling optimization problem in production process as an engine to drive digital twin. The performance of the whole line was evaluated and regulated by order simulation. Wang et al. [18] put forward the active scheduling mode based on the initiative of workpieces and machines, and discussed the interactive system, response mechanism, operation mode and mutual scheduling behavior of active scheduling. Leng $\mathrm{J}$ et al. [19] proposed an intelligent modeling method based on real-time perception and decision-making of service state of bottom manufacturing resources in CPS in order to meet the requirements of self-organizing allocation and adaptive collaborative control of manufacturing resources in intelligent factories. Ting $\mathrm{Qu}$ [20] et al. 
extended the concept of online control in UPU manufacturing by using the idea of digital twin, and put forward the idea, mechanism and quantitative optimization method of multi-system linkage optimization control. The method was applied into three stages of production process: precise planning, real-time tracking and dynamic control. The corresponding technology and system have been applied in leading enterprises such as Carbury Chemical Group. Starting from the workshop level, the basis of manufacturing activities, in order to gradually solve the interaction and integration between the physical world and the information world in the workshop level, and ultimately achieve the goal of intelligent production and precise service, the concept of digital workshop/factory was put forward many years ago. Scholars and practitioners [2125] at home and abroad have carried out a lot of research and construction work around digital workshop/factory, including workshop manufacturing association, digital/virtual workshop modeling, workshop data/information integration, workshop intelligent operation and precise management, etc. Many valuable theories and technologies have been put forward to analyze the problem and goal at workshop level from different angles, and put forward the corresponding solutions.

\section{Research gaps}

However, in the context of the new generation of information technology, there are still the following limitations and shortcomings in realizing the demand of intelligent manufacturing and production:

(1) the Internet of things in Workshop Manufacturing. Current research mainly focuses on the intelligent perception and access (including interfaces, protocols, and models), state and operation data acquisition/transmission/processing, operation status monitoring and health management of a single device. A large number of theoretical, technical and methodological studies have been carried out. At the same time, relevant devices have been developed. Therefore, it is said that the network access and intelligent operation of a single device have been realized to a certain extent. However, insufficient attention has been paid to the interconnection of heterogeneous elements in workshop (such as equipment and equipment, people and equipment, equipment and environment, people-equipment-environment, etc.). Especially, there is a lack of research on the system-level comprehensive interconnection of heterogeneous elements such as human, machine, material and environment, that is to say, there is a lack of the support of the theory of full interconnection and integration of heterogeneous elements in workshop and general devices.

(2) Digital/Virtual Workshop Modeling/ Simulation/ Analysis. Existing research mainly focuses on the construction and simulation analysis of geometric model or system model of workshop elements, lacking of models to depict and reflect the real behavior, rules and constraints of physical workshop, which makes it impossible to realize the real and complete mirror from physical workshop to virtual workshop. In addition, in the simulation analysis of workshop operation, existing research mainly relies on existing data-driven models. The factors such as workshop behavior, rules and constraints, as well as the integration of real-time dynamic data of workshop physical elements, are not considered enough, that is, the lack of support from the multi-dimensional fusion model of geometry-behaviorrules-constraints.

(3) Workshop Data/Information Integration. Integration and sharing of information/data related to workshop is mainly based on an information system or platform. Its integration scope is limited to the existing data and part of real-time data collected from information systems deployed in workshop (such as MES, enterprise resource planning, computer-aided process planning, etc.). The upstream business/process (such as market demand, product design) and downstream business/process (such as product maintenance, maintenance and overhaul data) of workshop are not considered and integrated adequately. In addition, due to the lack of support of intelligent interconnection and fusion theory and devices for heterogeneous elements in workshop, the collection and integration of real-time physical data in workshop is insufficient. Also, the two-way dynamic link and fusion of physical and information data are insufficient, that is, the lack of consideration and support for all-factor/allbusiness/all-process physical-information fusion data in workshop.

(4) Workshop operation optimization and precise management. Traditional ideas are mainly based on the process of 'Problem Analysis-Model ConstructionSolution Algorithms Design-Optimized Analysis Control'. After the Internet of Things and big data are applied into workshop, scholars at home and abroad have proposed a new idea of 'Data Association MiningDynamic Evolution-Simulation/Forecasting-Intelligent Control' based on big data of workshop. Both of them depend on the operation state model and data of workshop. In the process of actual operation optimization and precise management of digitized workshop, due to the lack of 'geometry-behavior-ruleconstraint' fusion model of workshop and consideration and drive of workshop physics-information fusion data, the information layer and physical layer of workshop are not synchronized, workshop information layer and physical layer are in poor consistency, low degree of intelligence, inadequate management precision and not synchronized. Moreover, there is no support of workshop application and service.

\section{The distributed CPS architecture of compressor manufacturing}

The distributed CPS architecture of compressor manufacturing, as show in figure 1, mainly includes industrial internet infrastructure layer, physical space entity model layer, virtual space multi-dimensional model layer, multi-dimensional model association and 
mapping layer, big data analysis and decision-making layer, and digital twin application layer.

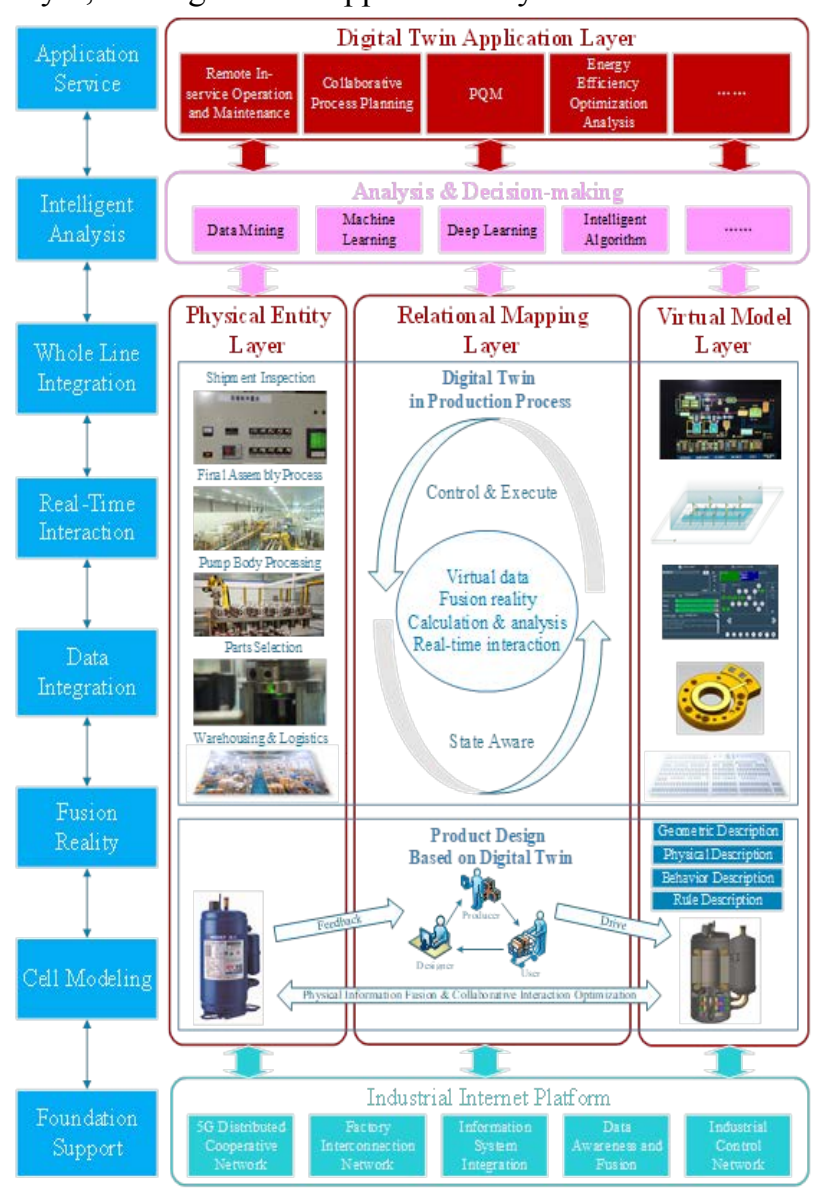

Fig.1. The distributed CPS architecture of compressor manufacturing.

The physical space entity layer includes total factor physical entities in products, equipment, workstations and production lines, such as raw materials, structure parts, assemblies, numerical control machines, robots, detection devices, and AGV. Whether it is the interactive integration of total factor physical resources, the simulation calculation of multi-dimensional virtual model, or the data analysis and processing, all of them are based on total factor physical entities. Meanwhile, the physical entities drive the operation of all parts, so that the digital twin could be realized, which is the 'skeleton' of the digital twin application.

The industrial internet infrastructure layer provides products and twin objects with unidirectional, bidirectional and one-to-many data synchronization and exchange, multi-space-time scale calculation, and closed-loop control capabilities for intelligent perception and interconnection of physical elements, realization of connection and interaction, real-time control and execution. It runs through the whole life cycle of products, which is the cornerstone of the application of digital twin system. The layout of industrial internet infrastructure in compressor assembly shop is shown in figure 2 .

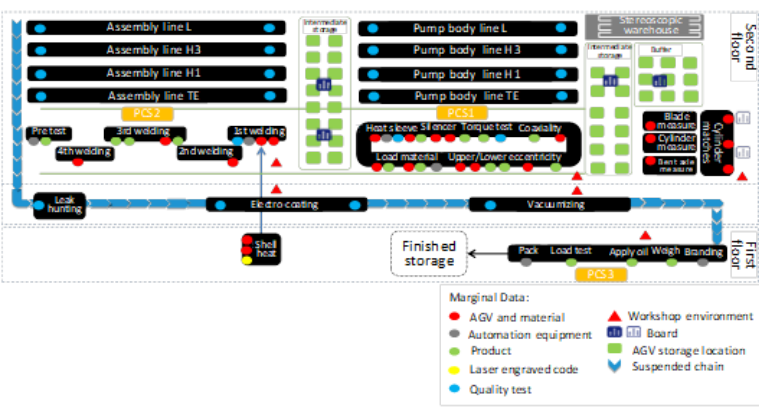

Fig.2. The layout of industrial internet infrastructure in compressor assembly shop.

The virtual space multi-dimensional model layer is used to describe the geometry, physics, behavior and rules of the above physical entities comprehensively. The geometric dimension describes geometric parameters such as size, shape and assembly relationship. The physical dimension analyses physical properties such as stress, fatigue and deformation, and the behavior dimension responds to external driving. The rule dimension is used to model the rule of the physical entity, so that the model has many functions, that is evaluation, optimization, prediction and testing. Multi-dimensional virtual model is the core component of various functions, such as product design, manufacturing, fault prediction, health management, etc. It is the 'heart' of digital twin application to transform application function from theory into reality with data-driven. The virtual space multidimensional model of compressor assembly is shown in figure 3 .

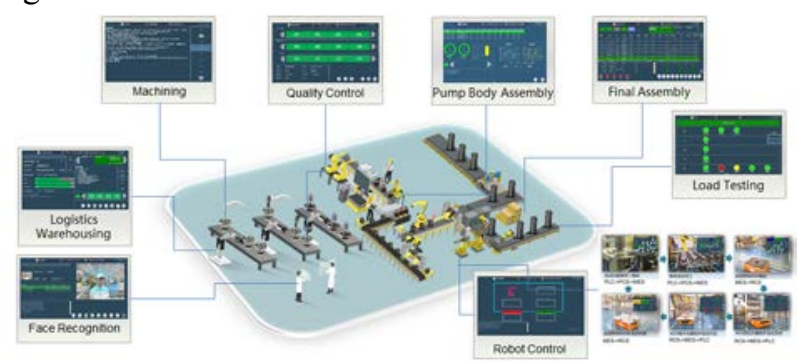

Fig.3. The virtual space multi-dimensional model of compressor assembly.

Multi-dimensional model association relation and mapping layer of physical space and virtual space is to sort out the data association relation of compressor manufacturing process, which processes industrial data of structured, semi-structured and unstructured compressor through unified data space-time dimensions such as timestamp, product number and equipment number and establish the twin data mainline of the whole life cycle with the key production factors of the production line, such as products, equipment, logistics and so on.it is used to realize the mapping of the whole life cycle of physical entity by virtual model and the symbiosis of virtual and real by combining with process flow of compressor production line and logistics route. There are various linear and non-linear, time-varying and time-invariant factors in the production process of compressors. Therefore, it is necessary to design the dynamic updating mechanism of physical entities' maintenance, upgrade and performance degradation, and 
establish the virtual evolutionary symbiosis neural network, to realize the mapping and motion simulation of physical entities, so that the dynamic updating capability of physical entities at each stage could always be achieved. The core element of digital twin is twin data, which originates from physical entity, virtual model and service system. At the same time, it is integrated into each part after fusion to promote the operation of each part. It is the 'blood' of digital twin application. Dynamic real-time interactive connection links physical entities, virtual models and service systems into an organic whole, so that information and data could be exchanged and transferred among different parts. It is the 'blood vessel' of digital twin application.

Based on existing historical data and specification data, data analysis decision-making layer uses deep learning technology such as deep neural network to establish intelligent prediction, analysis and decisionmaking model. Compressors' physical entity and its virtual model run synchronously. The generated twin data include not only real-time data-aware such as vibration, temperature, speed and current, but also simulation data such as fatigue wear, torsional vibration and thermal stress of shafting produced by virtual model, and fusion data obtained by multi-dimensional data processing and fusion. The Multi-dimensional model association relation and mapping layer of physical space and virtual space for compressor assembly is shown in figure 4.

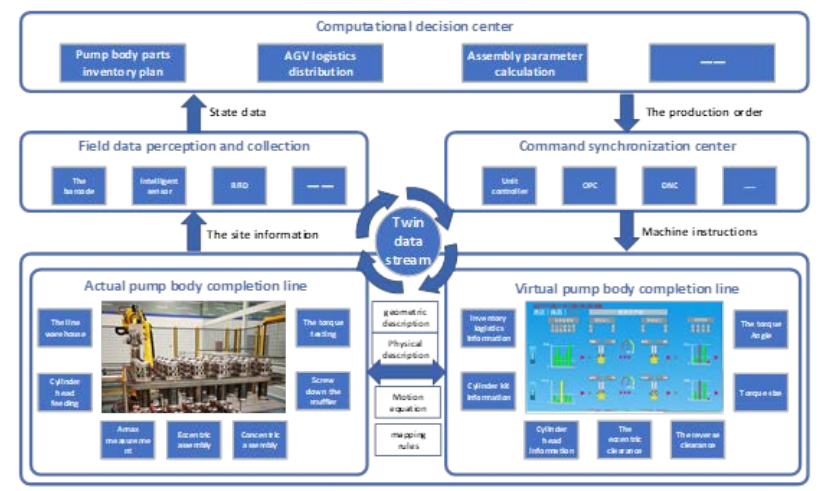

Fig.4. The Digital twin application for compressor quality detection.

In summary, establish the basic platform of industrial internet firstly. On this basis, set up the digital twin models of product and production line units from the physical space entity layer, virtual space multidimensional model layer, multi-dimensional model association relation and mapping layer of physical space and virtual space. Then, integrate the whole line digital twin model in the 'material-equipment-stationproduction line' order, and carry out thematic application development and system integration accordingly.

\section{Conclusion}

This paper proposes an intelligent manufacturing reference model from the three dimensions of "manufacturing with data and management". The contributions of this paper are as follows:
1.Considering the multi-source heterogeneous elements of the man-machine, machine, material, environment and other workshops, the physical layer of the full-element physical space is established, and the multi-dimensional model layer of the virtual space is constructed from the multiple dimensions of geometry, physics, behavior and rules. Then, the compressor manufacturing process data is combed to realize the mapping of the virtual model to the physical life cycle of the physical entity, and the dynamic real-time interactive connection transfers information and data.

2.The industrial Internet infrastructure layer runs through all stages of the product life cycle, and simultaneously realizes data processing and execution control of the compressor digital twinning system.

3.Using the deep learning technology like deep neural network to establish intelligent prediction, analysis and decision-making model for the existing historical data and specification data of the compressor.

4.For the orderly integration of the whole line digital twinning model according to the "material-equipmentstation production line", the subject application of monitoring, early warning, prediction and optimization is realized in the compressor digital application layer.

\section{References}

1. Conry R D. Compressor: U.S. Patent 5,857, 348(1999)

2. Boschert S, Rosen R. Digital twin-the simulation aspect (Mechatronic Futures, Cham, 2016)

3. Giannoulis D, Massberg M, Reiss J D. Digital dynamic range compressor design-A tutorial and analysis, Journal of the Audio Engineering Society 60(6), 399-408 (2012)

4. Castellanos I D, Stine J E. Compressor trees for decimal partial product reduction[C]/Proceedings of the 18th ACM Great Lakes symposium on VLSI. ACM, 107-110 (2008)

5. Prytz R, Nowaczyk S, Rögnvaldsson $\mathrm{T}$, et al. Predicting the need for vehicle compressor repairs using maintenance records and logged vehicle data, Engineering applications of artificial intelligence, 41, 139-150 (2015)

6. El Saddik A. Digital twins: The convergence of multimedia technologies, IEEE MultiMedia, 25(2), 87-92 (2018)

7. West T D, Blackburn M. Is Digital Thread/Digital Twin Affordable? A Systemic Assessment of the Cost of DoD's Latest Manhattan Project, Procedia computer science, 114, 47-56 (2017)

8. Fourgeau E, Gomez E, Adli H, et al. System engineering workbench for multi-views systems methodology with 3DEXPERIENCE Platform. the aircraft radar use case (Complex Systems Design \& Management Asia, Cham, 2016)

9. Vogel-Heuser B, Konersmann M, Aicher T, et al. Supporting evolution of automated material flow systems as part of CPPS by using coupled meta 
models, 2018 IEEE Industrial Cyber-Physical Systems (ICPS), 316-323 (2018)

10. Gray D, Bowes D, Davey N, et al. The misuse of the NASA metrics data program data sets for automated software defect prediction[C]//15th Annual Conference on Evaluation \& Assessment in Software Engineering. IET, 96-103 (2011)

11. Glaessgen E, Stargel D. The digital twin paradigm for future NASA and US Air Force vehicles[C]//53rd AIAA/ASME/ASCE/AHS/ASC Structures, Structural Dynamics and Materials Conference 20th AIAA/ASME/AHS Adaptive Structures Conference 14th AIAA, 1818(2012)

12. Schleich B, Anwer N, Mathieu L, et al. Shaping the digital twin for design and production engineering, CIRP Annals, 66(1), 141-144 (2017)

13. Tao F, Zhang M. Digital twin shop-floor: a new shop-floor paradigm towards smart manufacturing, Ieee Access, 5, 20418-20427 (2017)

14. Tao F, Cheng J, Qi Q, et al. Digital twin-driven product design, manufacturing and service with big data, The International Journal of Advanced Manufacturing Technology, 94(9-12), 3563-3576 (2018)

15. Negri E, Fumagalli L, Macchi M. A review of the roles of digital twin in cps-based production systems, Procedia Manufacturing, 11, 939-948 (2017)

16. Schleich B, Anwer N, Mathieu L, et al. Shaping the digital twin for design and production engineering, CIRP Annals, 66(1), 141-144 (2017)

17. Zhang H, Liu Q, Chen X, et al. A digital twin-based approach for designing and multi-objective optimization of hollow glass production line, IEEE Access, 5, 26901-26911 (2017)
18. Wang X, Shi Z, Zhang F, et al. Mutual trust based scheduling for (semi) autonomous multi-agent systems[C]//2015 American Control Conference (ACC). IEEE, 459-464 (2015)

19. Leng J, Zhang H, Yan D, et al. Digital twin-driven manufacturing cyber-physical system for parallel controlling of smart workshop, Journal of Ambient Intelligence and Humanized Computing, 10(3), 1155-1166 (2019)

20. Zhang K, Qu T, Zhou D, et al. IoT-enabled dynamic lean control mechanism for typical production systems, Journal of Ambient Intelligence and Humanized Computing, 10(3), 1009-1023 (2019)

21. Simpson T W, Williams C B, Hripko M. Preparing industry for additive manufacturing and its applications: Summary \& recommendations from a National Science Foundation workshop, Additive Manufacturing, 13, 166-178 (2017)

22. Salter J D, Campbell C, Journeay M, et al. The digital workshop: Exploring the use of interactive and immersive visualisation tools in participatory planning, Journal of environmental management, 90(6), 2090-2101 (2009)

23. Orchard S, Hermjakob H, Julian Jr R K, et al. Common interchange standards for proteomics data: Public availability of tools and schema. Report on the Proteomic Standards Initiative Workshop, 2nd Annual HUPO Congress, Montreal, Canada, 8-11th October 2003, Proteomics, 4(2), 490-491 (2004)

24. Li B, Hou B, Yu W, et al. Applications of artificial intelligence in intelligent manufacturing: a review, Frontiers of Information Technology \& Electronic Engineering, 18(1), 86-96 (2017)

25. Gao Q, Shi R, Wang G. Construction of intelligent manufacturing workshop based on lean management, Procedia CIRP, 56, 599-603 (2016) 